\title{
White dwarf seismology: Influence of the constitutive physics on the period spectra.
}

\author{
G. FONTAINE
}

Département de Physique, Lniversité de Montréal, C.P. 6128, succursale A, Montréal, Québec, HSC $9 J 7$, Canada.

P. BRASSARD

Département de Physique, Université de Montréal, C.P. 6128, succursale A, Montréal, Québec, H9C 9.J7, Canada.

\begin{abstract}
We present the results of numerical experiments aimed at demonstrating how the $g$-mode period spectra of pulsating DA white dwarfs depend on the various components of the input physics. We take advantage of recent developments on many fronts of physics (equation of state, opacity, convection) to compare the theoretical pulsation periods of models with different pieces of the constitutive physics, but with otherwise fixed values of their stellar parameters. This exercise is necessary to assess the reliability of the pulsation analyses of white dwarfs which have started to come out.

Nous présentons les résultats de simulations numériques pour déterminer comment les périodes de pulsation (type $g$ ) des étoiles naines blanches DA dépendent des différentes composantes de la physique constitutive. A cet effet, nous avons utilisé des résultats récents au niveau de la physique de base (équation d'état, opacité, convection) pour comparer les périodes de pulsation de modèles stellaires ayant des paramètres fixes, mais qui diffèrent au niveau de leur physique constitutive. Notre démarche est essentielle afin de pouvoir quantifier les premiers résultats d'analyses d'étoiles pulsantes qui commencent à être publiés.
\end{abstract}

\subsection{Introduction}

It is now well established that white dwarf stars become intrinsically variable during certain phases of their evolution. For the majority of them, the 
so-called DA white dwarfs (with atmospheres dominated by hydrogen), luminosity variations are observed when the stars have effective temperatures in the rather narrow interval $13,000 \mathrm{~K} \gtrsim T_{\text {eff }} \gtrsim 11,000 \mathrm{~K}$ (Wesemael et al. 1991). As a DA white dwarf cools, its hydrogen eventually recombines in the outermost layers, leading to a very significant increase of the opacity there and to the formation of a superficial convection zone. By the time such a star has cooled down to $T_{\text {eff }} \sim 13,000 \mathrm{~K}$, the superficial hydrogen convection zone has grown deep enough to be able to drive nonradial pulsation modes of the gravity $(g)$ type. It is not known why the excitation mechanism should become inoperative in more evolved, older DA white dwarfs with $T_{\text {eff }} \sim 11,000 \mathrm{~K}$ and cooler. The $g$-mode pulsations manifest themselves in terms of multiperiodic luminosity variations with typical periods in the range 100-1200 $\mathrm{s}$ and light curve amplitudes (in the optical domain) ranging from about $\sim 5$ millimagnitude for the smallest amplitude variable known to upward of 0.3 magnitude for the larger amplitude variables.

The existence of pulsating white dwarfs offers us the fascinating possibility of using them to test directly the predictions of stellar evolution theory. In this context, we recall that evolution theory suggests that the bulk of the mass of a typical isolated white dwarf should consist of carbon and oxygen (the exact proportions are still unknown because of uncertainties in the rates of helium thermonuclear burning). Also, DA white dwarfs must have retained some small amounts of hydrogen and helium in their outer layers, and it is believed that diffusion processes rapidly sort these elements out, leading to an onion-like, stratified structure with hydrogen floating on top of a helium mantle, itself floating on a core of heavier elements (presumed to be $\mathbf{C}$ and $\mathbf{O}$ ). Standard evolutionary calculations such as those of Iben \& Tutukov (1984) or Koester \& Schönbenner (1986) for example, make definite predictions as to the actual values of the hydrogen and helium remnant masses. What asteroseismology offers us is the potential for probing the internal structure of white dwarfs and, hence, testing the expectations of stellar evolution theory.

Of particular interest here is the fact that the chemical stratification of a white dwarf model leaves a characteristic signature on the period spectrum of the star (see, e.g., Brassard et al. 1992a). We can then hope to infer the vertical run of the chemical composition and, in particular, the thickness of the outer hydrogen layer in a DA star by comparing the observed periods with those of theoretical models. Moreover, as a white dwarf cools, its structure changes slightly, which results in the slow evolution of a given pulsation mode in that star (Winget, Hansen, \& Van Horn 1983). This gives us the possibility, through long-term observations, of determining the 
cooling timescale of a pulsating white dwarf by measuring the rate of period change of a pulsation mode. In turn, we can use this result to infer the core composition of a white dwarf since the cooling rate is essentially proportional to the mean atomic mass of the core material in such a star.

In the twenty-odd years since their discovery, enormous progress has been made in our understanding of the properties of pulsating DA white dwarfs. Key review papers narrating this progress have successively been presented by McGraw (1977), Robinson (1979), Winget \& Fontaine (1982), Van Horn (1984), Winget (1988), and Kawaler \& Hansen (1989). However, despite the advances reported in these papers, it has not been possible (until very recently) to exploit fully the potential of white dwarf seismology. Indeed, due to shortcomings in the quality of the observational data and the lack of sufficiently powerful theoretical tools, it has not been possible to unambiguously identify the pulsation modes in white dwarfs. Without mode identification, inferrences about the internal structure are not possible. This situation is changing, however, and, in a very recent effort, Brassard et al. (1993) have presented the first successful seismological analysis of a pulsating DA white dwarf, including complete mode identification. From their analysis, they have inferred a hydrogen layer mass $\log \left(M(\mathrm{H}) / M_{\star}\right) \equiv \log q(\mathrm{H}) \simeq-5.90_{-0.20}^{+0.10}$ and a helium layer mass $\log \left(M(\mathrm{He}) / M_{\star}\right) \equiv \log q(\mathrm{He}) \simeq-2.61 \pm 0.02$ in the pulsating white dwarf G117-B15A.

In order to assess the reliability of such determinations (and others to follow), it is essential to study the influence of the constitutive physics on the period distributions and rates of period change computed from theoretical stellar models. This can be done by comparing the pulsation properties of stellar models with fixed parameters, but computed with different sets of constitutive physics. Thanks to the recent advances made on several fronts in this area (many of which discussed at this Colloquium), such a comparative study is now possible for white dwarfs. In the spirit of this meeting, we thus present the results of our analysis of the effects of the input physics of the $g$-mode period spectra of representative models of pulsating DA white dwarfs.

\section{2 $G$-mode periods and constitutive physics}

To construct a stratified DA white dwarf model it is necessary to specify five basic parameters: (1) the effective temperature $T_{\text {eff }},(2)$ the surface gravity $g_{s}$ (or, equivalently, the total mass $M$ since the equation of state specifies the mass-radius relationship in a white dwarf), (3) the core com- 
position, (4) the mass contained in the helium mantle $q(\mathrm{He})$, and (5) the mass of the outer hydrogen layer $q(\mathrm{H})$. The stellar structure equations are then solved with the help of a given set of constitutive physics, of which we can distinguish four components. An equation of state must first be known; for white dwarfs, the equation of state is made of an analytic part to describe the ideal gas atmosphere (Saha equations), a tabular part to describe the partially-ionized, partially-degenerate, nonideal envelope, and a tabular part to describe the strongly interacting, completely ionized, dense Coulomb fluid of the core. The opacity of the material must also be known. In addition to the radiative opacity which determines the transport properties of the envelope material, an accurate description of the conductive opacity must also be available to describe the energy transfer in the degenerate core. A physical model for convection is also required to describe how much flux is transported by convection in the outermost hydrugen layers in a pulsating DA white dwarf. Finally, a physical model is equally required to describe the composition transition zones at the $\mathrm{H} / \mathrm{He}$ and $\mathrm{He} / \mathrm{C}$ interface regions.

To demonstrate how the constitutive physics affects the $g$-mode period spectra of stars, we first recall a well-known result of linear pulsation theory. In the asymptotic limit of high radial overtones $(k \gg 1)$, and for a chemically homogeneous and purely radiative model, it has been shown (Tassoul 1980) that $g$ modes belonging to the same value of $\ell$ have a uniform period spectrum with a constant period spacing given by

$$
P_{k+1, \ell}-P_{k, \ell}=\frac{1}{\sqrt{\ell(\ell+1)}} \Pi_{o}
$$

where $P_{k, \ell}$ is the period of a $g$-mode with radial order $k$ and spherical harmonic azimuthal index $\ell$, and $\Pi_{o}$ is an integral quantity which depends only on the structural properties of a star. This quantity is given by

$$
\Pi_{o}=\frac{2 \pi^{2}}{\int_{0}^{R} \frac{|N|}{r} d r}
$$

where $N$ is the so-called Brunt-Väisälä frequency (i.e., the local natural oscillation frequency of fluid elements when buoyancy is the restoring force) whose square can be written in the following convenient way

$$
N^{2}=\frac{g^{2} \rho}{P} \frac{\chi_{T}}{\chi_{\rho}}\left(\nabla_{\mathrm{ad}}-\nabla-\frac{1}{\chi_{T}}\left[\frac{\partial \ln P}{\partial \ln Y}\right]_{\rho, T} \frac{d \ln Y}{d \ln P}\right)
$$


where $g$ is the local gravity, $\rho$ is the density, $P$ is the pressure, $\chi_{T}=$ $(\partial \ln P / \partial \ln T)_{\rho}$ and $\chi_{\rho}=(\partial \ln P / \partial \ln \rho)_{T}$ are the usual $\operatorname{logarithmic}$ pressure derivatives with respect to temperature and density, respectively, $\nabla_{\mathrm{ad}}$ is the adiabatic temperature gradient, $\nabla$ is the actual temperature gradient, and $Y$ is the mass fraction of helium which uniquely specifies the local chemical composition in stratified models of white dwarfs with $\mathrm{H} / \mathrm{He}$ and $\mathrm{He} / \mathrm{C}$ transition zones.

Equations (1)-(3) illustrate clearly how the constitutive physics enters the computations of $g$-mode periods. First, it is obvious that the equation of state plays a major role through the various thermodynamic quantities which appear in the Brunt-Väisälä frequency. Second, the opacity plays an equally important (if indirect) role by specifying the temperature gradient $\nabla$ and by determining the structure paths followed by a given model in the $(\rho-T)$ plane. Third, even though equation (2) is valid only for purely radiative models, it is possible to generalize it to models with thin convection zones in which $N^{2}<0$. In that case, the integral must be carried out only over the radiative regions, and the main influence of convection is then to increase $\Pi_{o}$ as compared to a purely radiative model. Fourth, the way the composition transition zones are treated affects directly the third term in parentheses in equation (3). This term gives an important contribution to $N^{2}$ only in composition transition zones where $Y$ varies.

White dwarf stars are, of course, neither purely radiative nor chemically homogeneous. These circumstances lead, in fact, to $g$-mode period spectra which are highly non-uniform. Nevertheless, when allowance is made for the presence of a convection zone in the evaluation of $\Pi_{o}$ (as indicated above), this quantity still bears relevance to the pulsation periods of a white dwarf model as it now gives a fairly good estimate of the mean period spacing, $\left\langle P_{k+1, \ell}-P_{k, \ell}\right\rangle$, of an otherwise nonuniform period spectrum.

\subsection{Reference model}

We have constructed a standard, reference model (STD) with parameters $T_{\text {eff }}=12,500 \mathrm{~K}, \log g_{s}=8.0$, pure carbon core, $\log q(\mathrm{He})=-2.7$, and $\log q(\mathrm{H})=-6.1$. These parameters are comparable to those inferred by Brassard et al. (1993) for G117-B15A. We have used a new model generator code based on an Runge-Kutta scheme to calculate full static stellar models. We developed this code in order to be able to fine tune theoretical pulsation periods to the observations, a task that cannot be accomplished efficiently with a stellar evolution code. This new tool is briefly described by Brassard $\&$ Fontaine (these Proceedings). The reference model makes use of improved 
versions for the envelope equation of state of Fontaine, Graboske, \& Van Horn (1977; FGV) and the core equation of state of Lamb (1974; L). It uses the radiative opacity tables provided by the Los Alamos Opacity Library Program (Huebner 1980; LAO). Moreover, it uses conductive opacity fits (IH) which we developed by combining the Itoh et al. $(1983,1984)$ data at high densities with the Hubbard and Lampe data (1969) at low densities. Convection is treated with the so-called ML2 version of the mixing-length theory (Fontaine, Villeneuve, \& Wilson 1981). An important improvement was made with regard to the treatment of the composition transition zones. We took advantage of the development phase of the model code to incorporate a full and self-consistent description of diffusive equilibrium in the transition zones. All previous calculations have been based on approximate (and sometimes completely arbitrary) treatments of this important piece of physics (for the pulsation periods).

Figure 1 shows the run of the Brunt-Väisälä frequency of the standard model from the center $\left(\log q \equiv \log \left(1-M(r) / M_{\star}\right)=0\right)$ to the surface of the star. In addition to the rapid decrease of $N^{2}$ toward the interior due to increasing degeneracy, the Brunt-Väisälä frequency profile is characterized by two structures which are important from a pulsation point of view because they lead to significant nonuniformities in the period spectrum. The most important of these is the structure centered on $\log q \simeq-6$ which is caused by the changing chemical composition in the thin $\mathrm{H} / \mathrm{He}$ transition zone of our chemically-layered model (the third term in equation [3]). Compared to our earlier results based on an approximate treatment of the composition transition zones (Brassard et al. 1992a), we now find wider transition zones and, as a consequence, the weaker structure associated to the $\mathrm{He} / \mathrm{C}$ transition zone plays a much less significant role than before. In Fig. 1, the effects of the deeper $(\log q \simeq-2.7) \mathrm{He} / \mathrm{C}$ transition zone are practically invisible.

The second feature of interest in Fig. 1 is the well around $\log q \simeq-16$ caused by the presence of a thin hydrogen convection zone in the outermost layers in which $N^{2}<0$. Very much like problems in quantum mechanics, the structures around $\log q \simeq-6$ and -16 lead to partial reflection and transmission at their sites and modify considerably the propagation characteristics of the pulsation waves and, ultimately, the eigenperiods. Modes can be trapped or confined above or below these regions, leading to highly nonuniform period spectra. It should be said that, for the periods of interest, chemical layering at the $\mathrm{H} / \mathrm{He}$ interface is largely responsible for the nonuniformities in periods, and those bear primarily the signatures of the strength and location of the $\mathrm{H} / \mathrm{He}$ transition zone. The convection zone, be- 


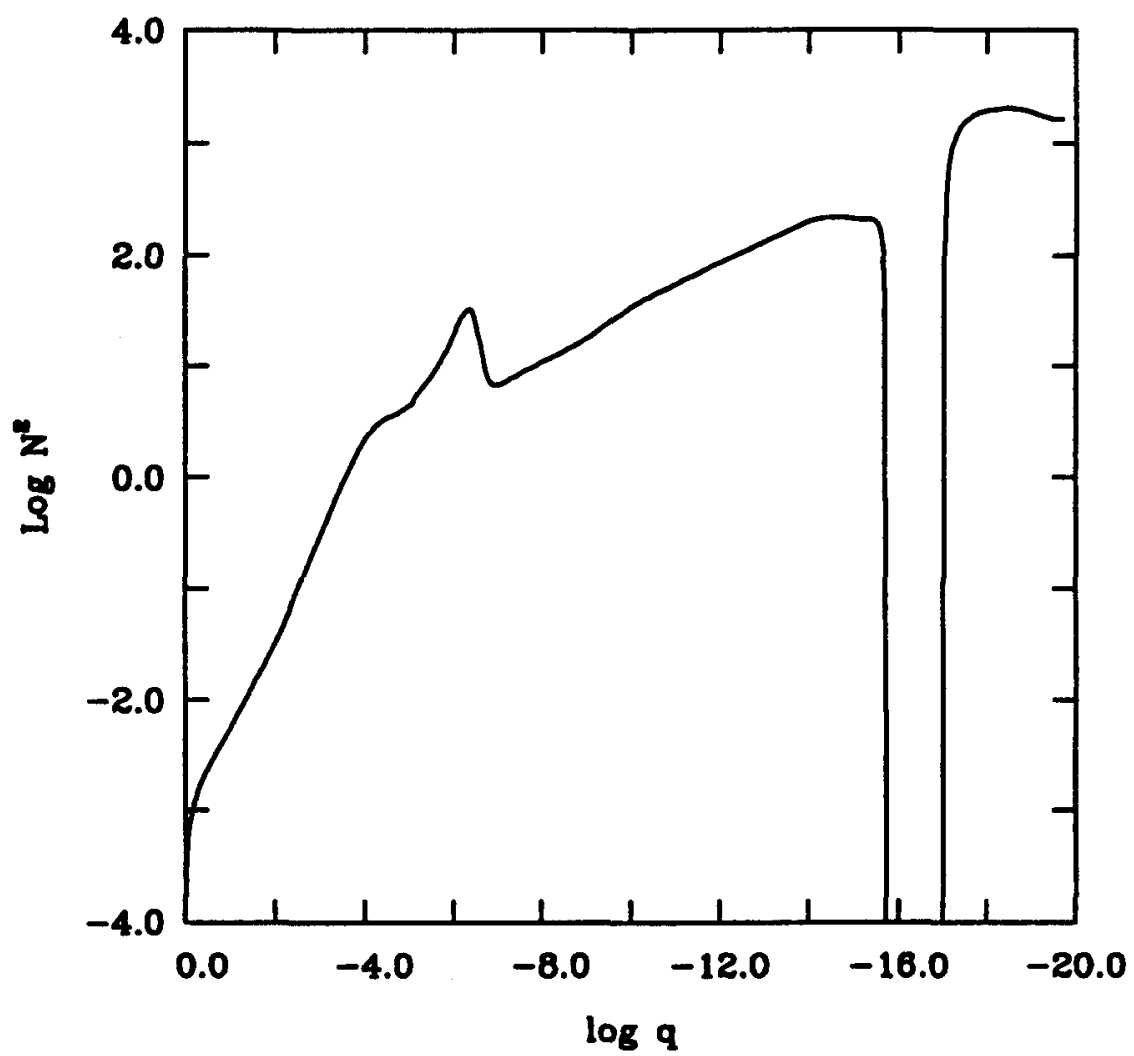

Fig. 16.1 Square of the Brunt-Väisälä frequency as a function of fractional mass depth in the reference model. Note that the choice of abscissa $\log q$ strongly emphasizes the outer layers; it is only in these outer regions that $g$ modes have nonnegligible amplitudes in white dwarfs.

cause of its position high in the envelope, can only affect high-order modes which have nodes reaching that high in the star.

Figure 2 shows the period spectrum of the standard model for $g$ modes with $\ell=2$ in the interval of periods from 100 to $1000 \mathrm{~s}$. The period distribution is shown here in a $\Delta P$ vs. $P$ diagram which is a plot of the period difference $\left(P_{k+1, \ell}-P_{k, \ell}\right)$ between two adjacent modes $(\Delta k=1)$ belonging to the same value of $\ell$ as a function of the period of the mode $\left(P_{k, \ell}\right)$ starting to the left with $k=1$. Very clearly, the period spectrum is not uniform, and this is due primarily to the presence of the $\mathrm{H} / \mathrm{He}$ transition zone near $\log q \simeq-6$. Nevertheless, we can still define an average period spacing which takes the value $28.99 \mathrm{~s}$ (as indicated by the horizontal dotted line). A direct integration over the model (eq. [2]) leads, on the other hand, 


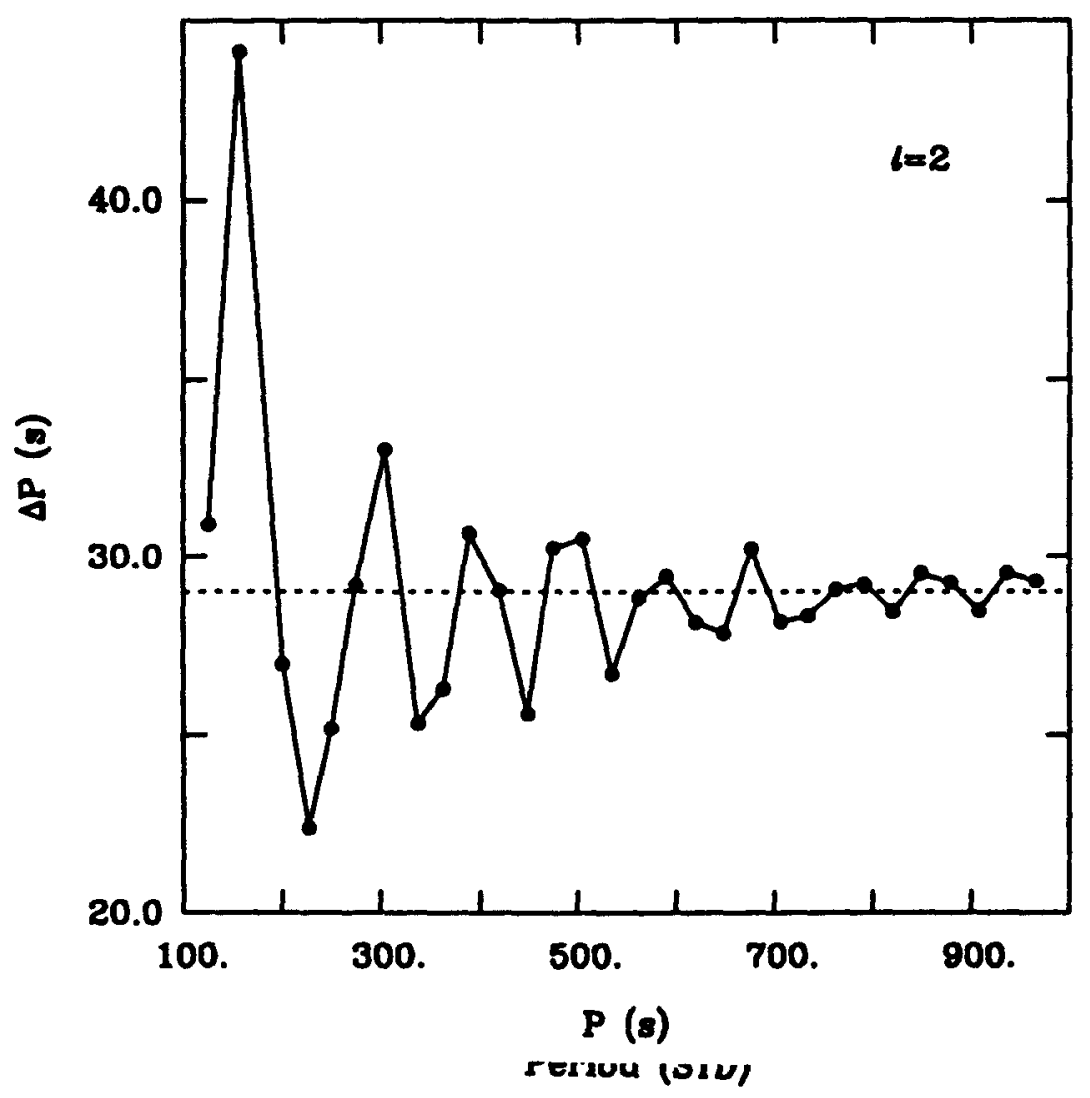

Fig. 16.2 Period spacing vs. period for modes with $\ell=2$ in the standard reference model.

to a value of $\Pi_{o}=75.74 \mathrm{~s}$, i.e., to an estimate of $75.74 / \sqrt{6}=30.92 \mathrm{~s}$ for the average period spacing for $\ell=2$ modes. This is close to the true number and indicates that, indeed, $\Pi_{o}$ is still of value for interpreting the pulsation periods of chemically stratified models. We will use this concept in what follows.

More insight can be obtained by examining, this time, the running integral $\int|N| / r d r$ as a function of depth in a model. Figure 3 shows the results for the standard model (continuous curve). The vertical dashed line marks the position of the degeneracy boundary, defined here as the layer where $\eta=5$ (where $\eta k T$ is the usual chemical potential for free electrons). The layer where the degeneracy parameter $\eta=5$ corresponds to the condition of approximate equality between the radiative and conductive opacities. We may note from Fig. 3 that more than $99.99 \%$ of the total mass of the standard model is located in the degenerate core. More importantly here, the 


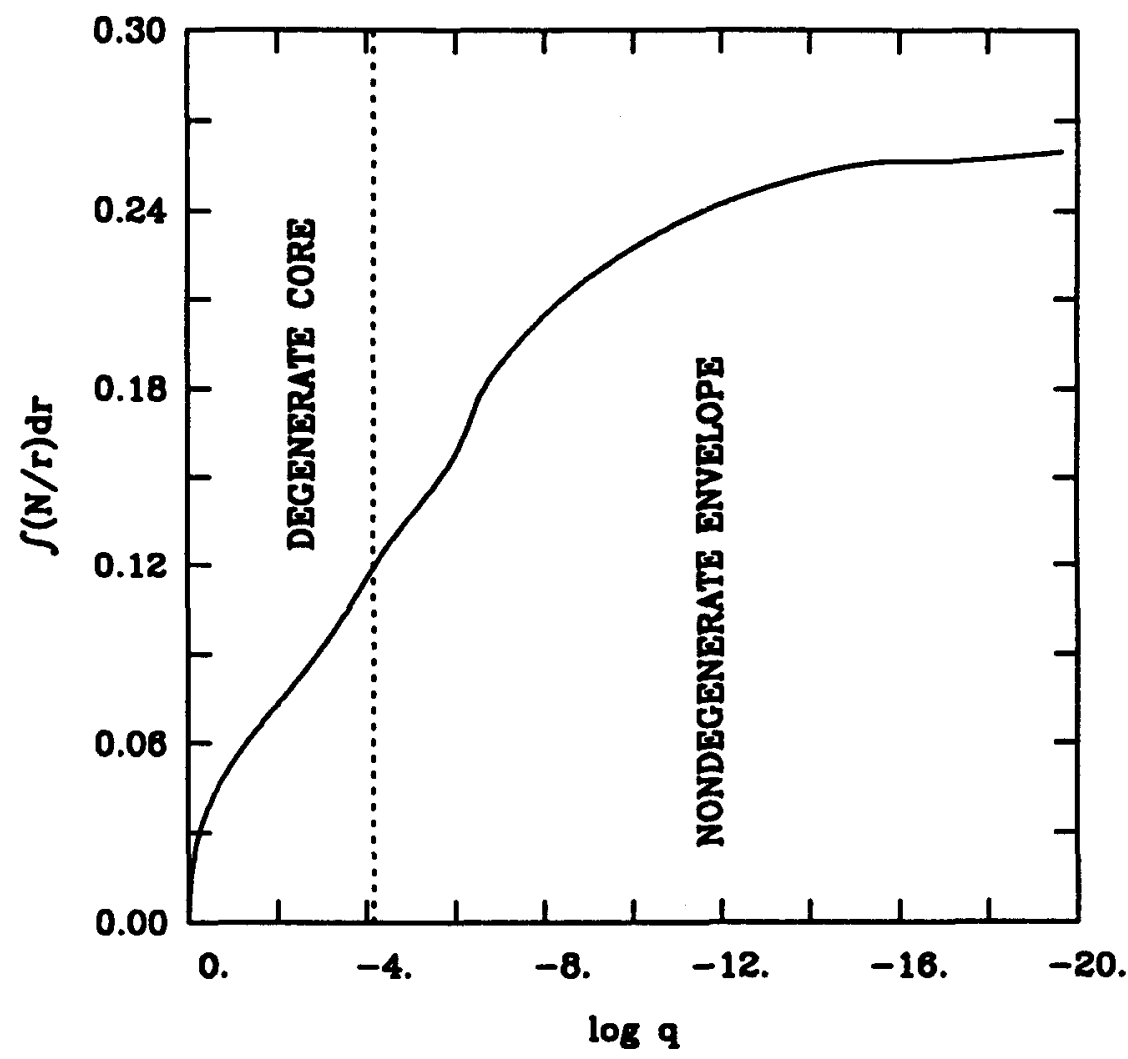

Fig. 16.3 Running integral $\int(|N| / r) d r$ vs. mass fraction from the center to the surface for the standard model. The vertical dashed line indicates the layer where the electron degeneracy parameter $\eta=5$.

figure shows that both the degenerate core and the nondegenerate envelope contribute roughly equally to the integral, which implies that the weight of period formation is distributed about equally between these two parts This result is of fundamental importance in the present context, and suggests that reliable constitutive physics must be available in the two regimes to derive reliable periods for white dwarfs.

\subsection{Comparative study}

We have calculated stellar models with the same parameters as those of the standard model but by varying one piece or another of the input physics. For each modified model, we next computed the $g$-mode periods for modes with $\ell=1,2$, and 3 in the period window $100-1000 \mathrm{~s}$ with the help of our 
Table 16.1. Parameters and input physics for the various equilibrium models

\begin{tabular}{|c|c|c|c|c|c|c|c|}
\hline Model & $\begin{array}{l}T_{\text {eff }} \\
(\mathrm{K})\end{array}$ & $\begin{array}{c}\text { Envelope } \\
\text { EOS }\end{array}$ & $\begin{array}{l}\text { Core } \\
\text { EOS }\end{array}$ & $\begin{array}{c}\text { Radiative } \\
\text { Opacity }\end{array}$ & $\begin{array}{c}\text { Conductive } \\
\text { Opacity }\end{array}$ & Convection & $\begin{array}{l}\Pi_{0} \\
(\mathrm{~s})\end{array}$ \\
\hline STD & 12,500 & FGV & L & LAO & IH & ML2 & 75.74 \\
\hline SC & 12,500 & SC & L & LAO & IH & ML2 & 75.78 \\
\hline IDEAL & 12,500 & Ideal & L & LAO & IH & ML2 & 73.68 \\
\hline NC & 12,500 & FGV & Ideal & LAO & IH & ML2 & 77.55 \\
\hline $2 \mathrm{~K}$ & 12,500 & FGV & L & $2 \times \mathrm{LAO}$ & $2 \times I H$ & ML2 & 68.22 \\
\hline HOT & 14,820 & FGV & L & LAO & IH & ML2 & 68.33 \\
\hline OPAL & 12,500 & FGV & L & OPAL & IH & ML2 & 76.73 \\
\hline CM & 12,500 & FGV & L & LAO & IH & CM & 75.73 \\
\hline
\end{tabular}

adiabatic finite element pulsation code (Brassard et al. 1992b). These are the values of interest for pulsating white dwarfs. We finally compared the periods with those of the standard reference model.

Table 1 summarizes the properties of the equilibrium models we used in our numerical experiments. The table includes the value of $\Pi_{o}$ obtained by direct integration of equation (2) for each model. This is a useful quantity to have because we can estimate from it how large should the period deviations be between two models. Neglecting the difference in periods for the first mode $(k=1)$ between two models, we find that, for $k>>1$, the expected period difference for the same mode (same $k$ ) between the two models should be

$$
P_{k, \ell}(2)-P_{k, \ell}(1) \simeq \frac{k-1}{\sqrt{\ell(\ell+1)}}\left(\Pi_{o}(2)-\Pi_{o}(1)\right) .
$$

We have used this last equation to obtain the results listed in Table 2 which apply to $\ell=2$ modes with a period $P \simeq 1000 \mathrm{~s}(k \simeq 31$ for the standard model). These expected period differences can be contrasted with the results of the detailed calculations. We discuss each of our numerical experiments in what follows.

Figure 4 summarizes part of our results for a first experiment in which the envelope equation of state has been varied. This is a plot of the period difference for the same mode (same value of $k$ ) computed, on the one hand, for a model in which the recent Saumon-Chabrier (SC; Saumon \& Chabrier $1989,1991,1992)$ equation of state for the $\mathrm{H} / \mathrm{He}$ envelope has been used and, on the other hand, for our standard model with the FGV equation 
Table 16.2. Expected period differences for $\ell=2$ modes at $P=1000 s$

\begin{tabular}{cc}
\hline \hline Models compared & $\Delta P(s)$ \\
\hline SC-STD & 0.5 \\
IDEAL-STD & -25.2 \\
NC-STD & 22.2 \\
2K-STD & -92.1 \\
2K-HOT & -1.3 \\
OPAL-STD & 12.1 \\
CM-STD & -0.1 \\
\hline \hline
\end{tabular}

of state, all other things being the same. The period difference is plotted in terms of the period of the standard model for the sequence of modes belonging to $\ell=2$. These results are quite typical of the other values of $\ell$ (the periods and period differences must be scaled by a factor $\sqrt{\ell(\ell+1)}$ ).

What the diagram shows is that the periods of the two models are quite similar with maximum relative deviations of $\sim 3 \%$. Typically, the differences are much less than that. The largest deviations occur for the low-order modes which are formed relatively deep in the star, and which are most sensitive to the thermodynamics of the helium plasma. The largest differences between the SC and the FGV data indeed arise for helium in the regime of interest for pulsating DA white dwarfs. Note that, even for hydrogen, there are substantial local differences in thermodynamic quantities between the two generations of equation of state tables (see the paper by Saumon in these Proceedings). However, as discussed above, pulsation modes are mostly sensitive to integrated properties, so that these local differences tend to average out. The very similar values of the integrals $\Pi_{o}(\mathrm{SC})=75.78 \mathrm{~s}$ and $\Pi_{\circ}(\mathrm{STD})=75.74 \mathrm{~s}$ (see Table 1 ) indeed reflect the fact that the pulsation properties of the two models must be very similar. The expected period difference of $0.5 \mathrm{~s}$ for the $\ell=2,1000 \mathrm{~s}$ mode (Table 2) is quite consistent with the actual difference shown in Fig. 4.

A happy consequence here, at least from our point of view, is that the newer SC equation of state data do not change significantly the period spectra of pulsating DA white dwarfs. The model parameters we have used in our experiments are typical of these stars, and varying the parameters within acceptable ranges will not change this conclusion. This means, in particular, that past calculations based on the FGV tables remain qualitatively and quantitatively reliable (see, e.g., Brassard et al. 1992a).

The reader should not be left with the impression that any envelope 


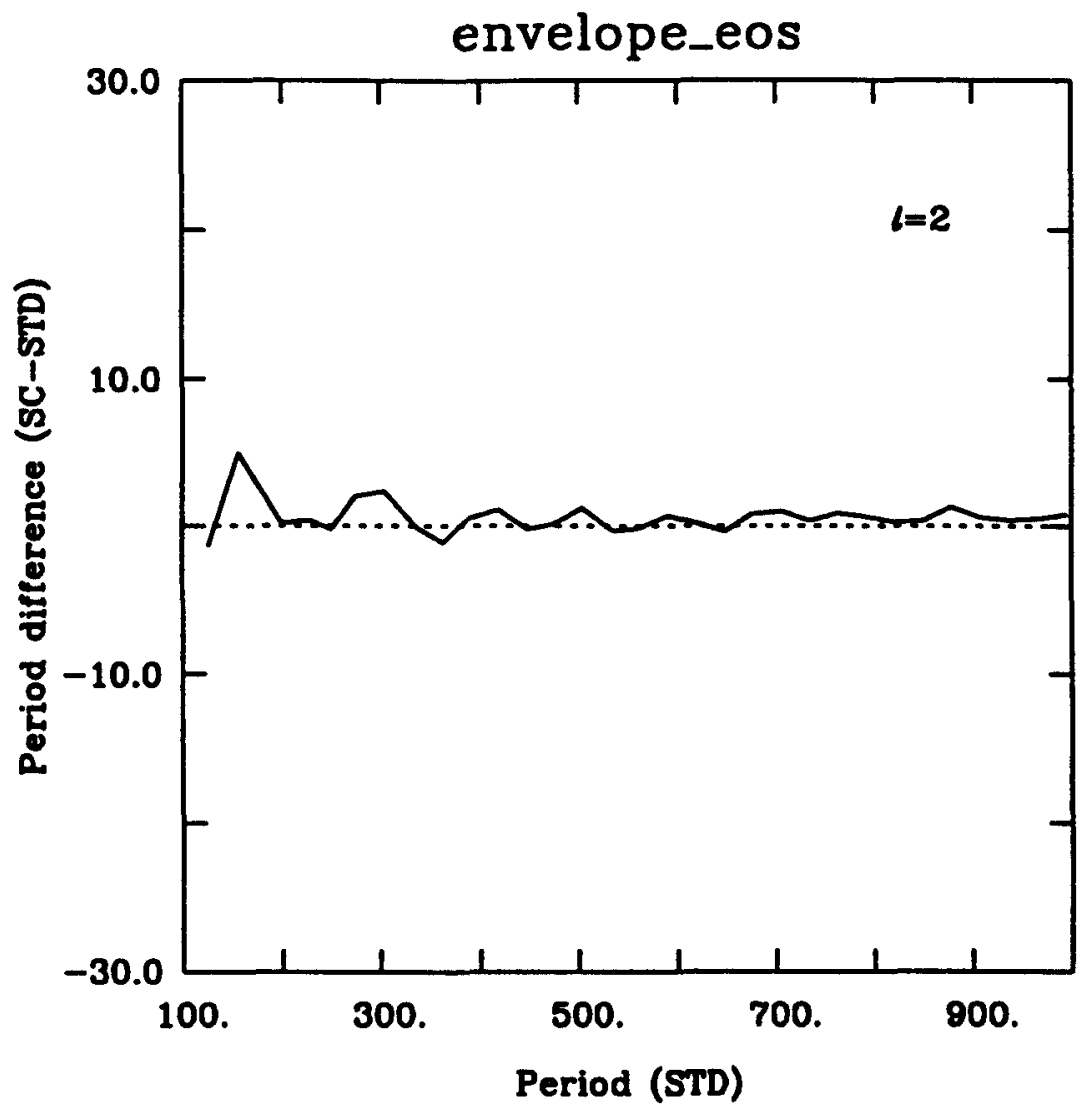

Fig. 16.4 Comparison of the pulsation periods of the SC and STD models. The figure shows the period difference of the two models for the same mode (same value of $k$ ) in terms of the period of the STD model. The results refer to $g$ modes belonging to the $\ell=2$ sequence.

equation of state is adequate to describe pulsating white dwarfs, however. In this context, we show, in Figure 5, some of the results of an experiment in which we have removed all nonideal terms and computed an envelope equation of state for pure $\mathrm{H}, \mathrm{He}$, and $\mathrm{C}$ on the basis of Saha equations only (IDEAL). Of course, as is well-known, this approach leads to complete recombination at sufficiently high densities, which is unphysical.

In a format identical to that of Fig. 4, the diagram shows period differences which are large compared to the previous results and which, moreover, are systematic. Indeed, the period difference grows systematically larger (in an absolute sense) with increasing radial order. The periods of the model based on the ideal equation of state become smaller than the periods of the standard model. For the $\ell=2,1000 \mathrm{~s}$ mode, the expected period difference 


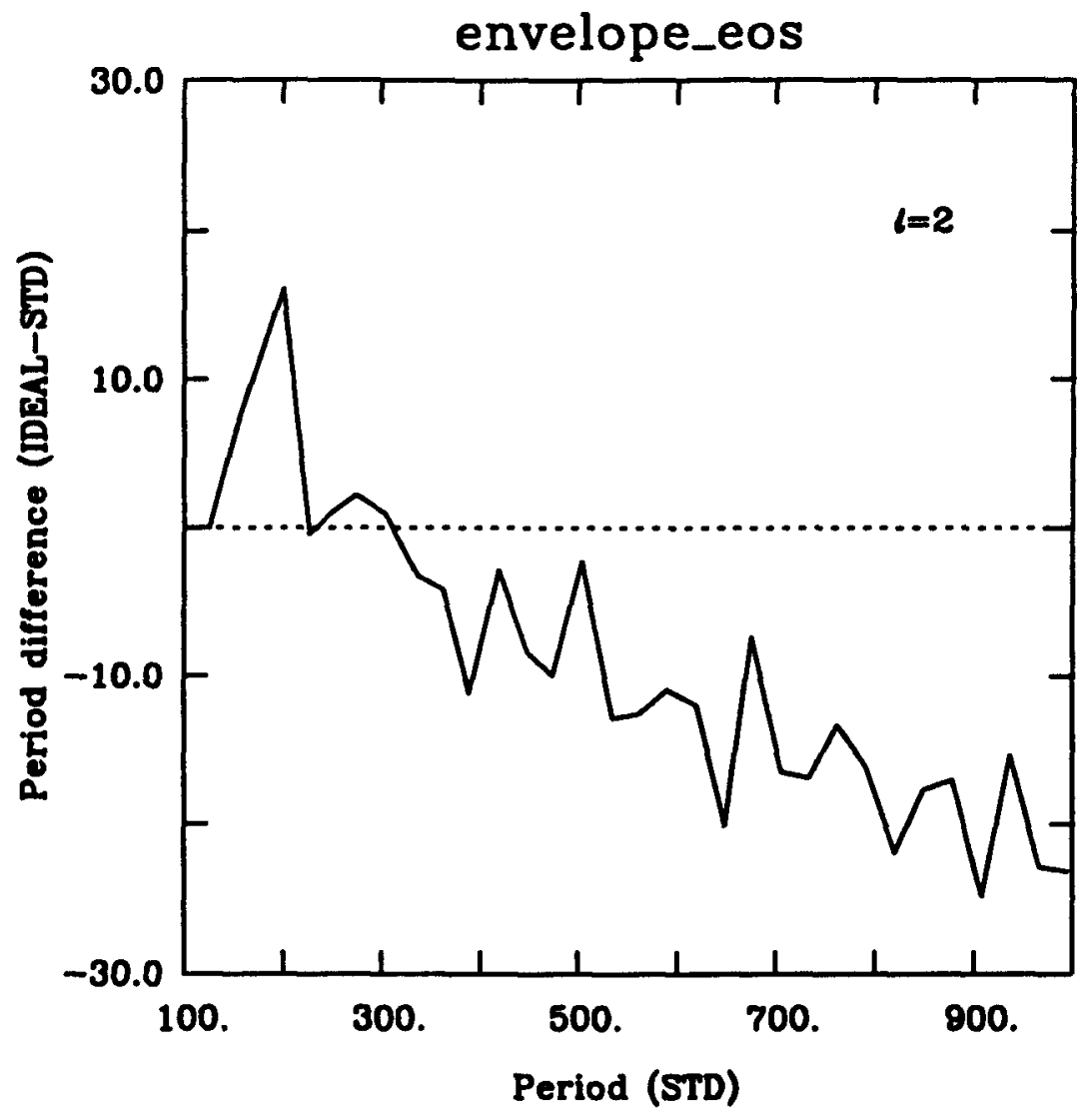

Fig. 16.5 Same as Fig. 4, but for the IDEAL and STD models.

is -25.2 s according to Table 2, which is comparable to the exact result shown in Fig. 5. Note also that, to a large extent, the jagged appearance of the curve is caused by mode trapping/confinement effects in the region where we rather crudely switch from our neutral, recombined ideal gas in the envelope to our totally ionized dense Coulomb fluid in the core.

We found it also interesting to investigate the effects of nonideal terms in the core equation of state. In the next experiment, we have thus removed all the nonideal terms in the pure $C$ equation of state of Lamb (1974; see also Lamb \& Van Horn 1975). This could be easily done because Lamb's code, among many nice features, is modular in its construction, so only the kinetic energy terms, for example, can be retained.

Figure 6 summarizes our results for the $\ell=2$ modes. The figure is on the same vertical scale as the previous ones, but there is a shift of the zero point. We can observe a systematic increase of the period with increasing radial order for the modified model (NC, which stands for "no Coulomb" terms) 


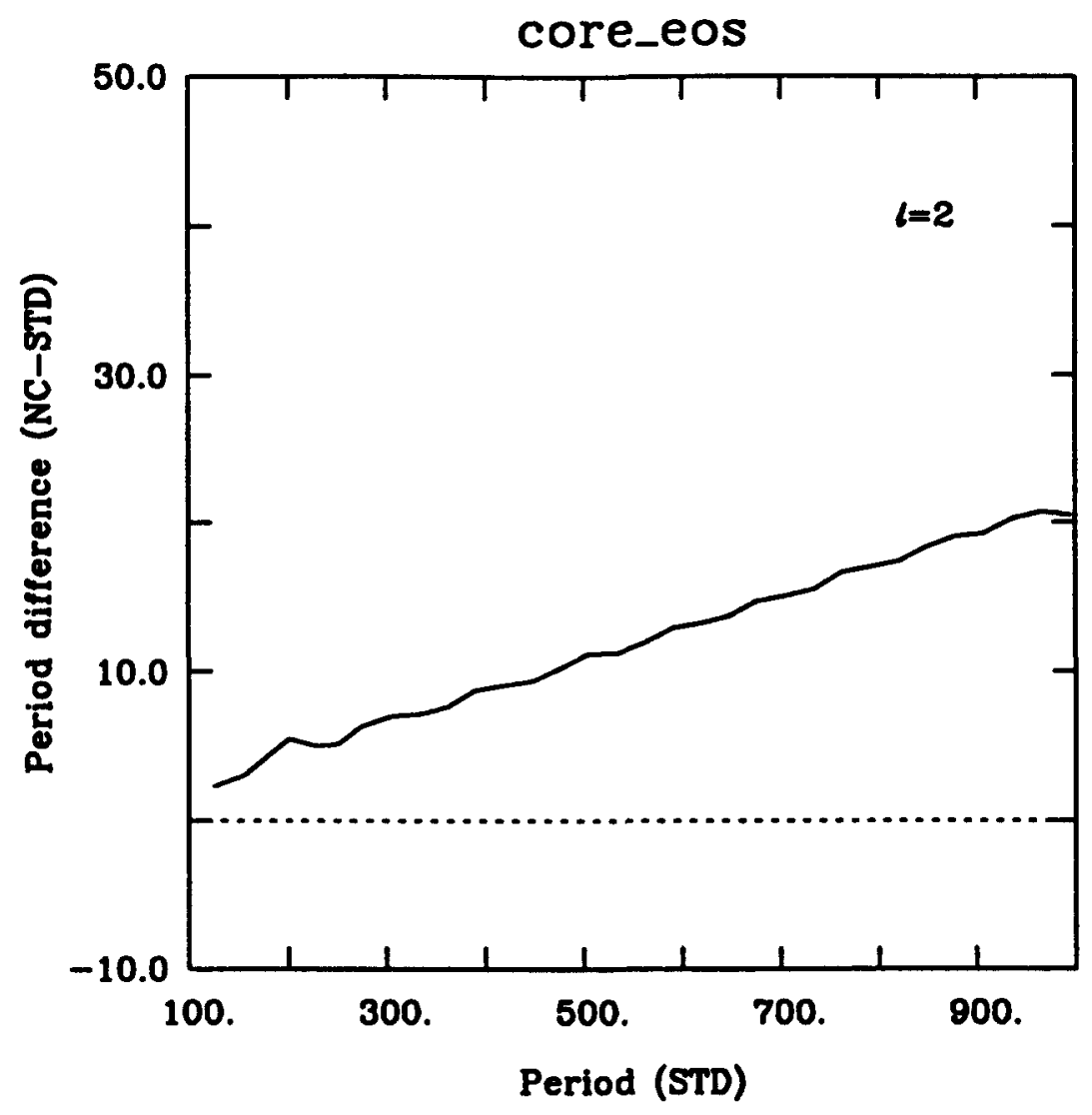

Fig. 16.6 Same as Fig. 4, but for the NC and STD models.

as compared to the reference model. The accumulated period difference at $1000 \mathrm{~s}$ is comparable to the expected value of $22.2 \mathrm{~s}$ (Table 2). We note that by keeping the surface gravity constant, the modified model can accommodate a larger mass than the standard model because the ideal NC core equation of state is harder. The upshot is that the altered model is slightly more degenerate in its interior, $|N|$ is smaller, $\Pi_{o}$ is larger, and the periods are larger than for our reference model.

We have carried out similar experiments with the opacity. For example, we have computed the period distributions of models in which the radiative opacity, the conductive opacity, and then the total opacity have been arbitrarily multiplied by a factor of 2 . The results for the experiment with the total opacity are illustrated in Figure 7 (modes with $\ell=2$ ). Again, the period difference is plotted in terms of the period of the standard model; the symbol " $2 \mathrm{~K}$ " here stands for the modified, more opaque model. Note that 


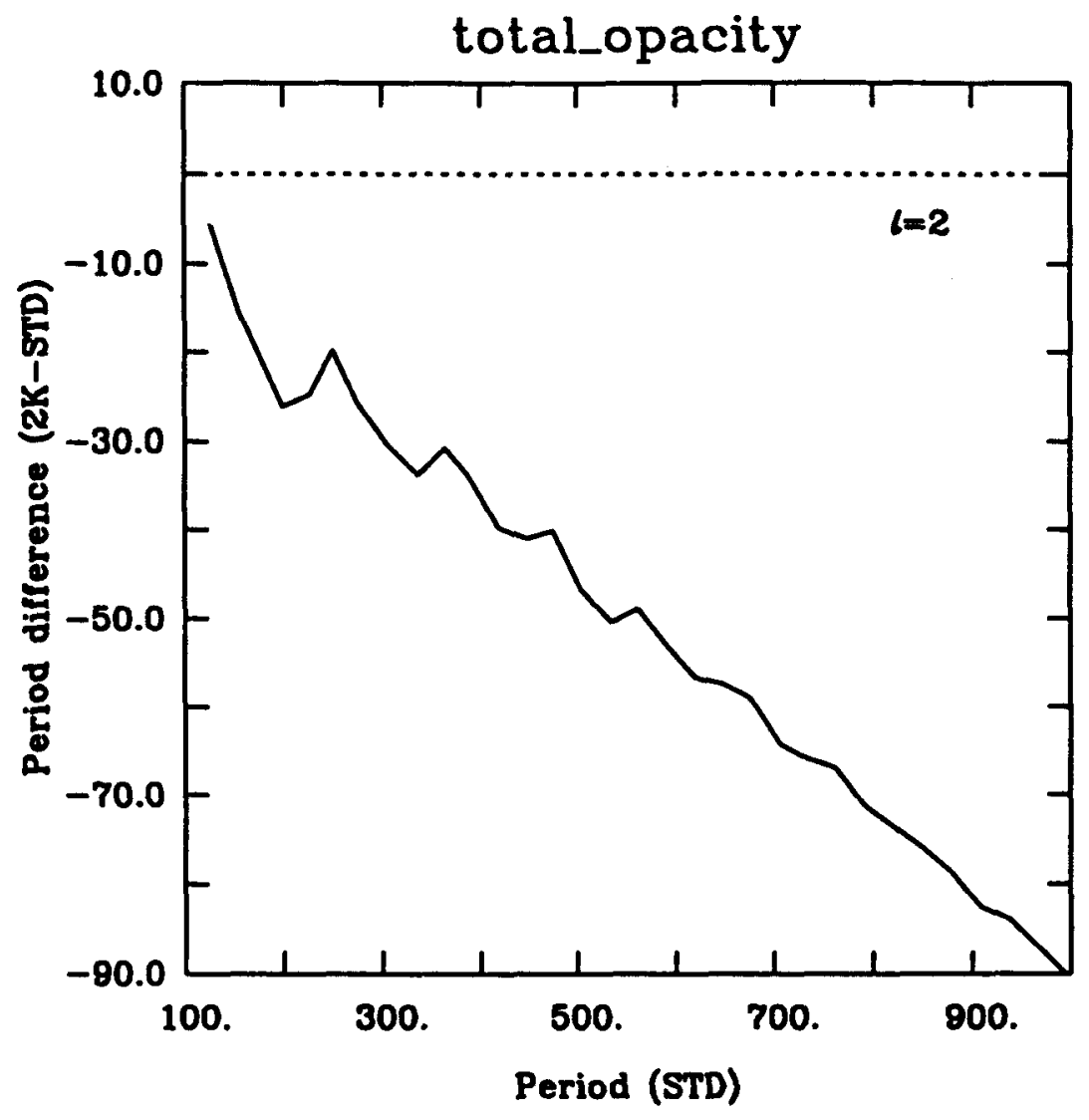

Fig. 16.7 Same as Fig. 4, but for the $2 \mathrm{~K}$ and STD models.

the vertical scale has been expanded to make allowance for the relatively large period differences.

We can observe that the $g$-mode periods of the opaque model are systematically smaller than those of the standard model. This is easily understood since an increase of the global opacity leads, for a model with a fixed surface temperature, to a larger core temperature. This, in turn, implies that the overall degeneracy of the core is less, the Brunt-Väisälä frequency is larger, and, consequently, the characteristic period spacing $\Pi_{0}$ is smaller in the modified model. We find indeed that the expected period difference of -92.1 s (Table 2) based on the $\Pi_{0}$ arguments match rather well the exact result for a mode with a period of $1000 \mathrm{~s}$.

It is interesting to point out that the more opaque model mimics very closely a hotter model. In other words, we can recover almost exactly the period spectrum of the more opaque model by considering a model with standard input physics, but with a higher effective temperature. Such a 


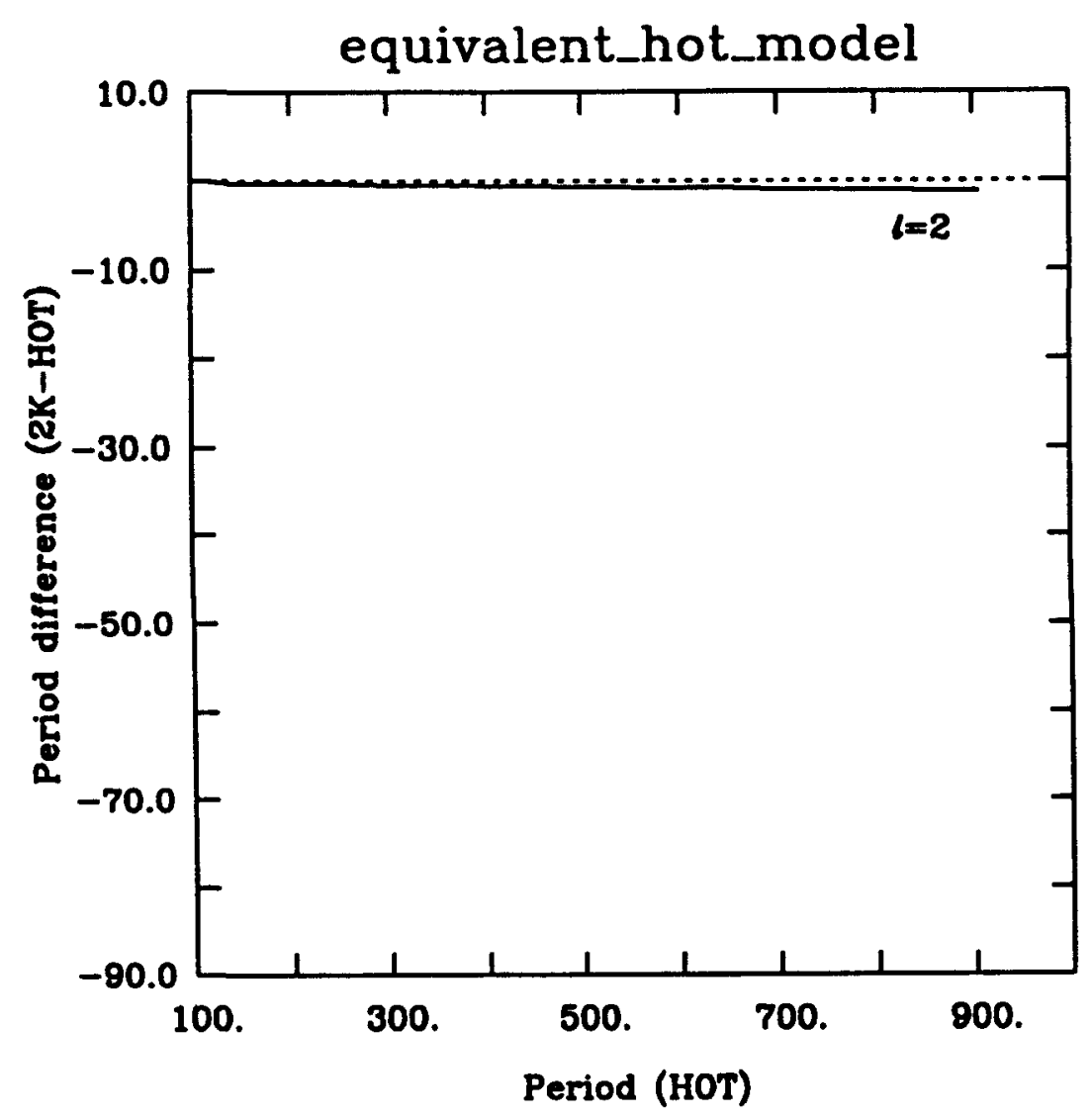

Fig. 16.8 Same as Fig. 4, but for the $2 \mathrm{~K}$ and HOT models.

model is referred to here as "HOT", and is the only equilibrium model for which we have changed a parameter ( $T_{\text {eff }}$ in the present case).

By trial and error, we have determined that a model with $T_{\text {eff }}=14,280 \mathrm{~K}$ has nearly the same central temperature as the opaque model at $T_{\text {eff }}=$ $12,500 \mathrm{~K}$, and a very similar value of the characteristic period spacing $\Pi_{0}$ (see Table 1). Accordingly, it is not surprising that the period spectra of the two models are nearly the same as illustrated in Figure 8 (plotted on the same scale as Fig. 7). A better agreement could have been found by fine tuning even more the effective temperature of the hot model, but we believe this is not necessary to make our point.

In another experiment, we have taken advantage of the recent availability of the OPAL Rosseland radiative opacity tables (Rogers \& Iglesias 1992) to compute a model (OPAL) incorporating the new data for pure $\mathrm{H}$, pure $\mathrm{He}$, and pure $C$. The periods of this model (for $\ell=2$ modes) are contrasted to those of the standard model in Figure 9. We find that the OPAL opacities 


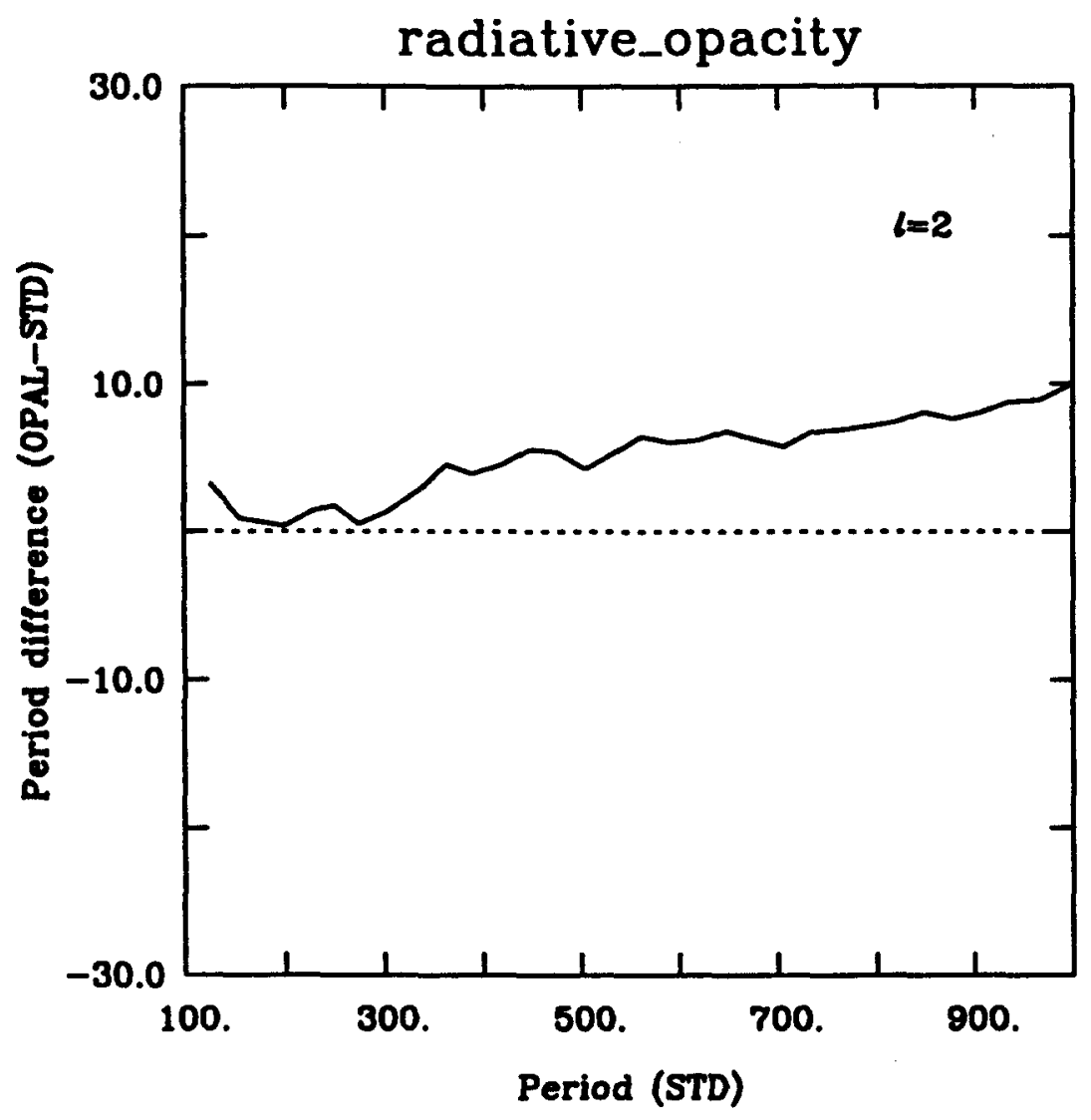

Fig. 16.9 Same as Fig. 4, but for the OPAL and STD models.

lead to systematically larger periods than the case based on LAO opacities. Again, the expected period difference of $12.1 \mathrm{~s}$ at $1000 \mathrm{~s}$ based on the integrals $\Pi_{o}$ is consistent with the result of Fig. 9. On the whole, we find that the OPAL opacities are slightly smaller than the LAO opacities in white dwarf envelopes, leading to models with slightly lower central temperatures and increased values of $\Pi_{o}$. We think that, in a large part, the differences are mainly caused by a difference in chemical composition: our older LAO tables refer to mixtures which contain small traces of heavy elements $\left(Z=10^{-3}\right)$, whereas we used the OPAL data for pure elements. We believe that the contribution of the $\mathrm{Z}$ elements to the LAO results makes the opacity somewhat larger, on the average, than the OPAL opacity (since the differences in opacity for pure $\mathrm{H}$ or pure $\mathrm{He}$, in particular, are not expected to be very significant between the two generations of tables). The test should probably be redone with radiative opacity tables referring to exactly the same chemical compositions. 
The effects of varying the convective efficiency using the ML1, ML2, and ML3 versions of the mixing-length theory have been thoroughly investigated by Brassard et al. (1992a), and will not be repeated here. We simply recall that an increase of the convective efficicency leads to a systematic increase of the $g$-mode periods. The effect is negligible for low-order modes but increases with increasing radial order.

We have recently incorporated in our model building code the parameterfree convection theory of Canuto \& Mazzitelli $(1991,1992 ; \mathrm{CM})$. At $T_{\text {eff }}=$ $12,500 \mathrm{~K}$, the CM model shows a hydrogen convection zone which is slightly less deep than the ML2 zone of the standard model. Since, at that effective temperature, the convection zone is quite high in the envelope (see Fig. 1), it does not contribute very much to the $\Pi_{o}$ integral. Accordingly, there is very little difference in the values of $\Pi_{o}$ for the CM and STD models (Table 1). Figure 10 illustrates that period differences only appear for the high-order modes, i.e., for those which have nodes reaching out into the high envelope and interacting with the convection zone. In the present case, the period differences are quite small. They would actually increase for cooler models (which develop deeper convection zones), but low-order modes would remain unaffected by convection.

Finally, we again refer the reader to Brassard et al. (1992a) for a discussion of the effects of varying the physical treatment used to describe the composition transition zones. In a nutshell, the main effect of, for example, decreasing the thickness of a composition transition zone is to increase the nonuniformities in the period spectrum; the average period spacing $\Pi_{o}$ remains largely unchanged.

\subsection{Discussion}

We have presented the results of a number of numerical experiments to illustrate how the $g$-mode period spectra of pulsating DA white dwarfs depend on the various components of the constitutive physics. In this exercise, we used stellar models with typical values for their parameters. In this connection, it should be reminded that, for reasons of distance, it is more complicated to read the asteroseismological records of pulsating stars than that of the Sun. For instance, when observing pulsating white dwarfs, we do not dispose a priori of independent and accurate estimates of basic stellar parameters such as total mass, total radius, surface gravity, effective temperature, and age. (We do not dispose either of thousands of well-identified modes!). Because these parameters are well known for the Sun, helioseismology can potentially be used to test the equation of state of the solar 


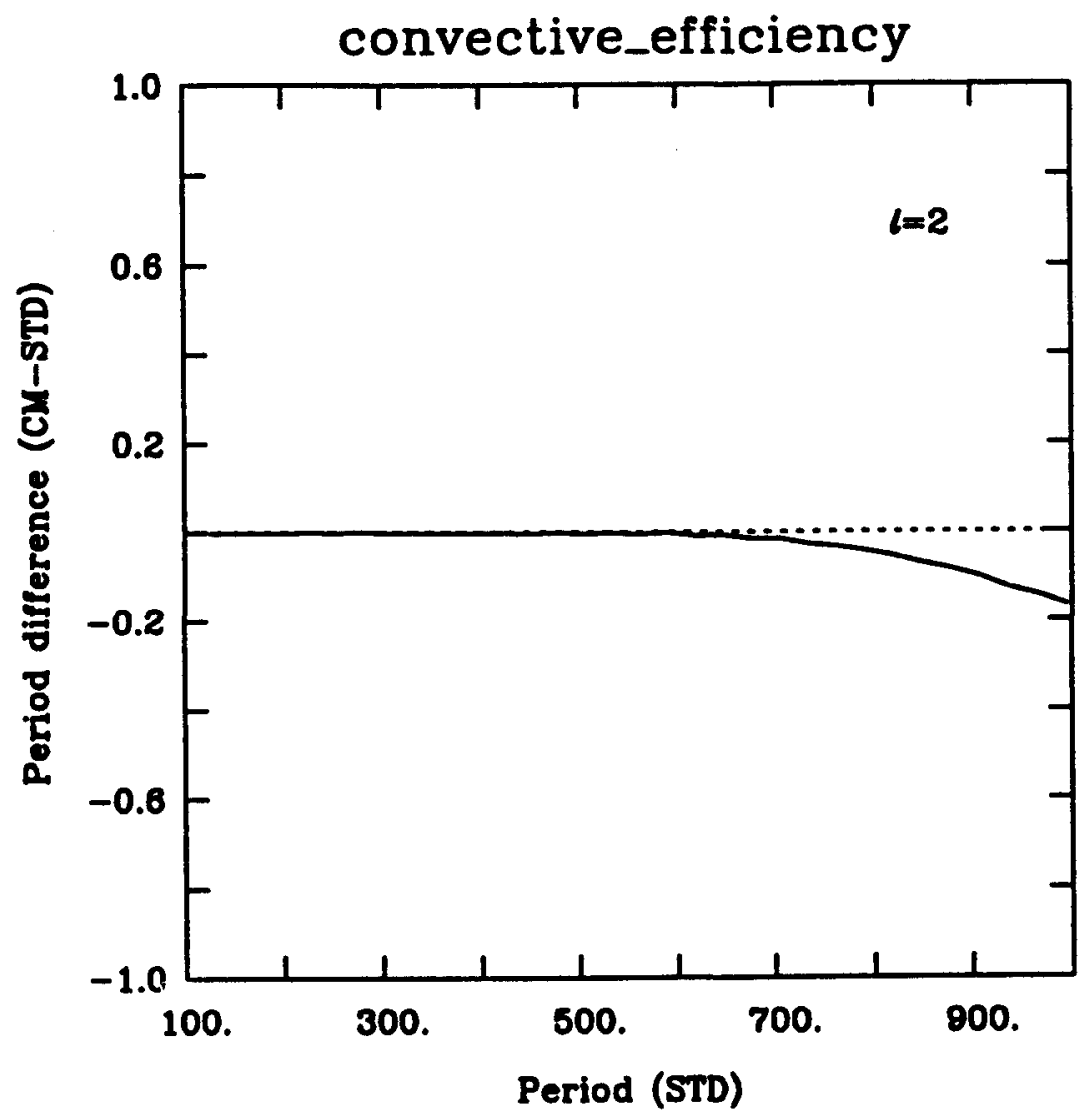

Fig. 16.10 Same as Fig. 4, but for the CM and STD models.

plasma as discussed by Däppen (these Proceedings). This is not a realistic claim to make for pulsating white dwarfs. In fact, what white dwarf seismology can really bring us are the means to infer the stellar parameters through the signatures they leave on the period distributions. In this approach, the stellar parameters are derived under the implicit assumption that the set of constitutive physics used in the model building phase is a good representation of the true physical conditions in the actual stars. The method we have used in this paper remains the only avenue for estimating the "internal errors" due to the constitutive physics. We note that Brassard et al. (1993) used a similar approach in their analysis of G117-B15A, and were able to show that their error budget is dominated by observational uncertainties (and not by shortcomings in the constitutive physics).

We conclude by pointing out that the constitutive physics bears on two more aspects of white dwarf seismology, which could not be discussed in this paper due to space shortage. While we have focussed here on the influence 
of the input physics on the period spectra, the question of rates of period change is also quite important. These rates depend, of course, directly on the cooling rate, a problem which has received considerable new attention in recent years, and which has been reviewed by Chabrier, Garcia-Berro, and Mazzitelli at this Colloquium. We note that in the range of effective temperature where the pulsating white dwarfs are found, no "exotic" mechanism (neutrino cooling, crystallization, phase separation in the core, etc.) are expected to dominate, so the computed cooling rates across the narrow instability strip should be quite reliable.

Finally, there is the question of mode excitation in pulsating white dwarfs. Nonadiabatic pulsation calculations by several independent groups all indicate that the driving region is located near the base of the hydrogen convection zone in pulsating DA white dwarfs. The question of mode driving and, in particular, the question of the location of the theoretical blue edge of the instability strip boil down to how deep is the hydrogen convection zone in a given model. Clearly, the most important component of the constitutive physics in the present context is the physical model used to describe convection. (The envelope equation of state as well as the radiative opacity also bear on the problem). A nonadiabatic survey to assess the influence of different convection models on the question of the location of the theoretical blue edge is a timely project. We have undergone such a project (Brassard, Fontaine, \& Wesemael 1993).

\section{Acknowledgement}

We wish to thank friends and collaborators for providing invaluable support, input, and insight during the course of our ongoing investigations of pulsating white dwarfs. Special thanks are due to those who have provided the basic data used in this paper: Gilles Chabrier, Hal Graboske, Bill Hubbard, Walter Huebner, Naoki Itoh, Don Lamb, Italo Mazzitelli, Forrest Rogers, Didier Saumon, and Hugh Van Horn. This work was supported in part by the NSERC Canada and by the Fund FCAR (Québec).

\section{References}

Brassard P., Fontaine G., Wesemael F., \& Tassoul M. Ap. J. Suppl. 81, 747, (1992a)

Brassard P., Pelletier C., Fontaine G., \& Wesemael F. Ap. J. Suppl. 80, 725, (1992b)

Brassard P., Fontaine G., \& Wesemael F., in preparation, (1993)

Brassard P., Fontaine G., Bergeron P., Wesemael F. \& Vauclair G., in preparation, (1993) 
Canuto V., \& Mazzitelli I. Ap. J. 370, 295, (1991)

Canuto V., \& Mazzitelli I. Ap. J. 389, 724, (1992)

Fontaine G., Graboske H.C. Jr. \& Van Horn H.M. Ap. J. Suppl. 35, 293, (1977)

Fontaine G., Villeneuve B., \& Wilson J. Ap. J. 243, 550, (1981)

Hubbard W.B. \& Lampe M. Ap. J. 163, 297, (1969)

Huebner W.F., private communication, (1980)

Iben I. Jr., \& Tutukov A.V. Ap. J. 282, 615, (1984)

Itoh N., Mitake S., Iyetomi H. \& Ichimaru S. Ap. J. 273, 774, (1983)

Itoh N., Kohyama Y., Matsumoto N. \& Seki M. Ap. J. 285, 758, (1984)

Kawaler S.D., \& Hansen C.J., IAU Colloq. 114, White Dwarfs, ed. G. Wegner (New York:Springer-Verlag), 97, (1989)

Koester D., \& Schönberner D. Astr. Ap. 154, 125, (1986)

Lamb D.Q., Ph. D. thesis, University of Rochester, (1974)

Lamb D.Q. \& Van Horn H.M. Ap. J. 200, 306, (1975)

McGraw J.T., Ph. D. thesis, University of Texas at Austin, (1977)

Robinson E.L., IA U Collog. 59, White Dwarfs and Variable Degenerate Stars, eds. H.M. Van Horn \& V. Weidemann (Rochester: University of Rochester Press), 343, (1979)

Rogers F.J. \& Iglesias, C.A. Ap. J. Suppl. 79, 507, (1992)

Saumon D. \& Chabrier G. Phys. Rev. Letters 62, 2397, (1989)

Saumon D. \& Chabrier G. Phys. Rev. A 44, 5122, (1991)

Saumon D. \& Chabrier G. Phys. Rev. A 46, 2084, (1992)

Tassoul M. Ap. J. Suppl. 43, 469, (1980)

Van Horn H.M., Proc. 25th Liège Astrophysical Colloquium, Theoretical Problems in Stellar Stability and Oscillations, eds. A. Noels \& M. Gabriel (Liège:Université de Liège), 307, (1984)

Wesemael F., Bergeron P., Fontaine G. \& Lamontagne R.L., Proc. Seventh European Workshop on White Dwarfs, eds. G. Vauclair \& E.M. Sion (NATO ASI Series), 159, (1991)

Winget D.E., IAU Symp. 123, Advances in Helio- and Asteroseismology, eds. J. Christensen-Dalsgaard \& S. Frandsen (Dordrecht:Reidel), 305, (1988)

Winget D.E. \& Fontaine G., Pulsations in Classical and Cataclysmic Variable Stars, eds. J.P. Cox \& C.J. Hansen (Boulder: University of Colorado Press), 46, (1982)

Winget D.E., Hansen C.J. \& Van Horn H.M., Nature 303, 781, (1983) 